\title{
Unsteady viscous MHD flow over a permeable curved stretching/shrinking sheet
}

\begin{abstract}
Purpose $\ddot{i}$ The purpose of this paper is to theoretically study the problem of the unsteady boundary layer flow past a permeable curved stretching/shrinking surface in the presence of a uniform magnetic field. The governing nonlinear partial differential equations are converted into ordinary differential equations by similarity transformation, which are then solved numerically.

Design/methodology/approach $\ddot{i}$ The transformed system of ordinary differential equations was solved using a fourth-order Runge-Kutta integration scheme. Results for the reduced skin friction coefficient and velocity profiles are presented through graphs and tables for several sets of values of the governing parameters. The effects of these parameters on the flow characteristics are thoroughly examined.

Findings $i ̈$ Results show that for the both cases of stretching and shrinking surfaces, multiple solutions exist for a certain range of the curvature, mass suction, unsteadiness, stretching/shrinking parameters and magnetic field parameter.

Originality/value $\ddot{i}$ The paper describes how multiple (dual) solutions for the flow reversals are obtained. It is shown that the solutions exist up to a critical value of the shrinking parameter, beyond which the boundary layer separates from the surface and the solution based upon the boundary layer approximations is not possible.
\end{abstract}

Keyword: Boundary layer; MHD flow; Unsteady; Curvature; Stretching/shrinking sheet 